\author{
Military Technical College \\ Kobry El-kobbah, \\ Cairo, Egypt
}

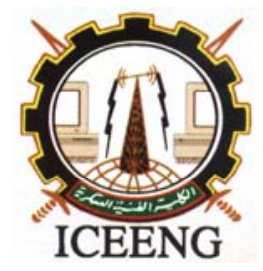

\title{
SHIELDING PERFORMANCE OF SUPERCONDUCTING STRUCTURES
}

\author{
Mohamed A H Eleiwa, Ph.D. \\ The Egyptian Armed Forces
}

\begin{abstract}
Electromagnetic shielding is one of the most promising applications of superconductors that still needs further investigation. An enhanced model is therefore proposed to design optimum shielding structures made from different low and high criticaltemperature (LTS \& HTS) superconductors. The proposed model incorporates classical electrodynamics with both superconductivity macroscopic theories such as two-fluid model, Ginzburg-Landau (GL) theory, and microscopic Bardeen-Cooper-Schrieffer (BCS) theory. Thermodynamic properties are also included using improved Gorter-Casimir relations based on published experimental data. General shielding effectiveness expressions are derived for different superconducting shielding structures against generally polarized incident electromagnetic waves. A computer algorithm is then developed to investigate the impact of superconducting material parameters on shielding effectiveness. The limitations and advantages of superconducting shielding structures are also discussed.
\end{abstract}

\section{KEY WORDS}

Superconductors, Shielding Effectiveness, and Computer Modeling.

\section{INTRODUCTION}

The discovery of High-Temperature Superconductivity (HTS) at liquid nitrogen temperature $(77 \mathrm{~K})$, coupled with recent advances in cryogenic technology has created major opportunities for the significant commercial realization of superconducting electronic devices. Applications [1] currently being pursued are mostly extensions of current technology used with the Low-Temperature Superconductors (LTS) such as powerful magnets used in Magnetic Resonance Imaging (MRI) scanners. Additional applications include microwave communication devices, waveguides, antenna systems [2], more efficient power transmission lines [3], smaller and more efficient generators, energy storage devices, particle accelerators, and levitating trains. Magnetic shielding is also one of the important applications of superconductors [4]. 
Electromagnetic screening (shielding) [5] is one of the key techniques for protection against illumination from both external fields and electromagnetic leakage from electronic products. Variety of shielding techniques are needed and play crucial roles in either electromagnetic compatibility or electromagnetic susceptibility ( EMC / EMS) compliance testing or system design. Previous studies and practices on shielding [4-5] solved many practical problems, but there are still more that present special challenges to engineers concerned with EMI/EMC. Shielding of static or low-frequency (LF) magnetic fields is a topic receiving growing interest in the EMC community due to various motivations. A reason relies upon the consideration that the traditional shielding approach of using conductive and / or high-permeability materials is not efficient. A new reason is the concern about risks associated to the exposition of population to LF electromagnetic fields. Ever since the superconductivity was found out early this century, the superconductor, as a sort of shielding materials, has always been paid great attention to for its magnetism-resistant quality. However very strict conditions must be fulfilled to maintain super-conductive state, and superconductor shielding [6-7] is very limited in theoretical studying and practicing. When high criticaltemperature $\left(\boldsymbol{T}_{c}\right)$ superconductor was found out in 1986, another trend started in the whole world studying superconductivity theory and application. The liquid nitrogen refrigerant of the high temperature superconductor facilities is cheaper and easier to handle than liquid helium. So the shielding materials of superconductor can easily be obtained. Some experiments have been done recently in this field [8], but the superconductor shielding theory still needs improving.

In this paper, several aspects of the superconductor shielding design parameters are further discussed and analyzed. First, the electromagnetic properties of superconductors are derived using a proposed model based on experimental data, two-fluid model, Gorter-Casimir relation, microscopic Bardeen-Cooper-Schrieffer (BCS) theory, and Ginzburg-Landau (GL) macroscopic theory. The developed model is then applied to investigate the variations of different high and low- $\boldsymbol{T}_{\boldsymbol{c}}$ superconductors parameters versus temperature and frequency. Secondly, different shielding structures made from LTS and HTS superconducting materials are proposed and analyzed. The electromagnetic shielding effectiveness is derived, and the shielding parameters are determined for optimum shielding design. Finally, shielding effectiveness for different superconducting structures are compared with those of traditional (metal) shielding structures, and hence the excellent performance of superconducting materials as effective shielding structures against electromagnetic waves, especially at low frequencies, is demonstrated.

\section{ELECTROMAGNETIC MODELING OF SUPERCONDUCTORS}

According to the two-fluid model [9], the total current density $\overrightarrow{\boldsymbol{J}}$ in the superconductor is the sum of the normal conduction current density $\overrightarrow{\boldsymbol{J}}_{\boldsymbol{n}}$ (normal fluid), and the superconducting component $\overrightarrow{\boldsymbol{J}}_{\boldsymbol{s}}$ (super fluid). Under the effect of an external (sinusoidal-time varying) electric field $\overrightarrow{\boldsymbol{E}}$, the normal conduction electrons (each with mass $\boldsymbol{m}$ and charge $\boldsymbol{q}$ ) will experience collision and thus move with velocity $\overrightarrow{\boldsymbol{v}}$ given by Drude classical model as

$$
\vec{v}=\frac{q \tau}{m(1+j \omega \tau)} \vec{E}
$$


where $\tau$ is the carrier scattering time. The net (normal) current density $\overrightarrow{\boldsymbol{J}}_{\boldsymbol{n}}$ is found from the responses of all $\boldsymbol{n}_{\boldsymbol{n}}$ carriers as

$\overrightarrow{\boldsymbol{J}}_{\boldsymbol{n}}=\boldsymbol{n}_{\boldsymbol{n}} \boldsymbol{q} \overrightarrow{\boldsymbol{v}}$

Substituting equation (2) into equation (1) to get the well-known Ohm law:

$\vec{J}_{n}=\frac{n_{n} q^{2} \tau}{m(1+j \omega \tau)} \vec{E} \nRightarrow \sigma_{n} \vec{E}$

where $\sigma_{n}=\frac{n_{n} q^{2} \tau}{\boldsymbol{m}(1+j \omega \tau)}$ is the normal alternating current (ac) conductivity. On the other hand, the charge carriers in the superconductors; namely Cooper pairs (super fluid) with density $\boldsymbol{n}_{\boldsymbol{s}}$, are not subjected to a drag force (perfect conductor). Such a condition is equivalent to $\tau \rightarrow \infty$, when substituting into equation (3) yields the super fluid current density $\overrightarrow{\boldsymbol{J}}_{\boldsymbol{s}}$ as :

$\vec{J}_{s}=\frac{n_{s} q^{2}}{j \omega m} \vec{E}=\sigma_{s} \vec{E}$

Equation (4) can be rewritten in time domain (the usual form of first London equation) as

$\frac{\partial \vec{J}_{s}}{\partial t}=\frac{\vec{E}}{\mu_{o} \lambda_{L}^{2}}$

where $\lambda_{L}=\sqrt{\frac{m}{\mu_{o} q^{2} n_{s}}}$ is known as London penetration depth

The first London equation models the superconductor as a perfectly conducting medium and does not account for magnetic flux expulsion (Meissner effect). Therefore the second London equation is derived by taking the curl of both sides of equation (5) and combining Faraday law to give

$\nabla \times \overrightarrow{\boldsymbol{J}}_{s}=-\frac{\overrightarrow{\boldsymbol{B}}}{\mu_{o} \lambda_{L}^{2}}$

The complex conductivity of the superconductor $(\sigma)$ is then derived by adding equations (3) and (4) to get the total current density $\overrightarrow{\boldsymbol{J}}$ as

$\overrightarrow{\boldsymbol{J}}=\overrightarrow{\boldsymbol{J}}_{n}+\overrightarrow{\boldsymbol{J}}_{s} \equiv \sigma \overrightarrow{\boldsymbol{E}}=\left(\sigma_{1}-j \sigma_{2}\right) \overrightarrow{\boldsymbol{E}}$

from which, the general expression for $\sigma$ is obtained as

$$
\sigma=\sigma_{1}-j \sigma_{2}=\frac{n_{n} q^{2} \tau}{m\left(1+\omega^{2} \tau^{2}\right)}-j\left(\frac{n_{s} q^{2}}{\omega m}+\frac{n_{n} q^{2} \omega^{2} \tau^{2}}{\omega m\left(1+\omega^{2} \tau^{2}\right)}\right)
$$

substituting the total current density $\overrightarrow{\boldsymbol{J}}$ into the following Maxwell equation:

$$
\nabla \times \vec{H}=j \omega \varepsilon_{o} \vec{E}+\vec{J} \equiv j \omega \varepsilon_{o} \varepsilon_{r} \vec{E}
$$

Then the complex relative permittivity $\varepsilon_{\boldsymbol{r}}$ may be written in the following form: 


$$
\varepsilon_{r}=\varepsilon^{\prime}-j \varepsilon^{\prime \prime}=\left(1-\frac{n_{s} q^{2}}{\omega^{2} m \varepsilon_{o}}-\frac{n_{n} q^{2} \omega^{2} \tau^{2}}{\omega^{2} m \varepsilon_{o}\left(1+\omega^{2} \tau^{2}\right)}\right)-j\left(\frac{n_{n} q^{2} \tau}{\omega m \varepsilon_{o}\left(1+\omega^{2} \tau^{2}\right)}\right)
$$

Once $\varepsilon_{r}$ is obtained, the complex wave number $\boldsymbol{k}$, the propagation constant $\gamma$, and the intrinsic impedance $\eta$ for an electromagnetic wave propagating in a superconducting medium are then derived by substituting equation (10) into $k=\omega \sqrt{\mu_{o} \varepsilon_{o} \varepsilon_{r}}, \gamma=j k=\alpha+j \beta$, and into $\eta=\sqrt{\frac{\mu_{o}}{\varepsilon_{o} \varepsilon_{r}}}$. The surface impedance $Z_{s}$ can now be accurately obtained using the general definition of [10] as $\boldsymbol{Z}_{s}=\gamma / \sigma \equiv \boldsymbol{R}_{s}+\boldsymbol{j} \omega \boldsymbol{L}_{s}$, where $\boldsymbol{R}_{s}$ and $\boldsymbol{L}_{s}$ are the surface resistance and surface inductance; respectively.

To derive the temporal and spatial distribution of the magnetic flux density $\overrightarrow{\boldsymbol{B}}$ inside any linear, isotropic, and homogeneous superconductor, take the curl of both sides of equation (9), which is the full form of Ampere law and combining the result with the rest of Maxwell equations and the constitutive laws given by the above equations to get

$$
\left[\left(1+\tau \frac{\partial}{\partial t}\right)\left(1-\lambda_{L}^{2} \nabla^{2}+\mu_{o} \varepsilon \lambda_{L}^{2} \frac{\partial^{2}}{\partial t^{2}}\right)+\mu_{o} \sigma_{o} \lambda_{L}^{2} \frac{\partial}{\partial t}\right] \vec{B}=0
$$

To account for the temperature dependence of superconductor parameters, the following empirical relations are proposed by [11] as

$$
\begin{array}{ll}
n_{s}\left(T_{n}\right)=n_{o}\left(1-T_{n}{ }^{x}\right), & n_{n}\left(T_{n}\right)=n_{o}-n_{s}\left(T_{n}\right), \\
\lambda_{L}\left(T_{n}\right)=\frac{\lambda_{L}(0)}{\sqrt{1-T_{n}^{x}}}, & \tau\left(T_{n}\right)=\tau(1)\left[T_{n}{ }^{-1}+y\left(T_{n}{ }^{-x}-1\right)\right]
\end{array}
$$

where $\boldsymbol{T}_{\boldsymbol{n}}=\boldsymbol{T} / \boldsymbol{T}_{\boldsymbol{c}}$ is the normalized temperature $\boldsymbol{T}$ with respect to the critical temperature $\boldsymbol{T}_{c}$, and $\boldsymbol{n}_{\boldsymbol{o}}$ is the total electron density. The coefficients $x \sim(1.3-4)$ and $y \sim(1-20)$ are determined based on measured data for superconducting materials [12]. When $\boldsymbol{x}=\boldsymbol{4}$ and $\boldsymbol{y}=\boldsymbol{0}$, the temperature dependence proposed by Gorter-Casimir model is obtained.

\section{SHIELDING EFFECTIVENESS OF SUPERCONDUCTING STRUCTURES}

A general expression of the electromagnetic shielding factor is derived for a planar superconducting structure due to both absorption and reflection losses. Oblique incidence with either parallel or perpendicular polarization waves are considered. Also, the shielding factors against static magnetic fields are derived for spherical shells made from magnetic materials and superconductors.

\section{A. Electromagnetic Shielding Effectiveness Calculations}


As the wave penetrates the shield material with conductivity $\sigma$, permeability $\boldsymbol{\mu}$, permittivity $\boldsymbol{\varepsilon}$, and thickness $\boldsymbol{t}$, the amplitudes of both $\overrightarrow{\boldsymbol{E}}$ and $\overrightarrow{\boldsymbol{H}}$ fields decrease exponentially with attenuation factor $\boldsymbol{\alpha}$. Such attenuation process is known as absorption loss caused by the shielding structure, denoted by $\boldsymbol{A}$, and expressed in decibels [dB] by

$$
A=20 \log \left(\frac{E_{o}}{E_{o} e^{-\alpha t}}\right)=8.686 \alpha t
$$

Due to impedance mismatch, the incident wave from a medium with intrinsic impedance $\boldsymbol{\eta}_{1}$ is also reflected by the reflection coefficient $\Gamma$ at the shield surface, whose intrinsic impedance is $\boldsymbol{\eta}_{2}$, producing reflection loss $\mathrm{R}$ given by

$$
R=-20 \log \left|\frac{E_{\text {ref }}}{E_{\text {inc }}}\right|=-20 \log |\Gamma|
$$

Neglecting losses due to multiple reflections, the attenuation factor due to reflection and absorption for a plane wave approaching perpendicularly (TEM) a slab with thickness $\boldsymbol{t}$, and intrinsic impedance $\eta_{2}=\eta$, placed in free space $\left(\eta_{1}=\eta_{o}\right)$, is given by the ratio of the amplitude of transmitted to incident fields [5] as

$$
\frac{\left|E_{\text {transmittd }}\right|}{\left|E_{\text {incident }}\right|}=e^{-\alpha t} \frac{4 \eta_{o} \mid \eta \eta}{\left|\eta_{o}+\eta\right|^{2}}
$$

The shielding factor $(\mathrm{S})$ in decibels is then defined as

$$
S=-20 \log \left\{\frac{\left|E_{\text {transmitted }}\right|}{\left|E_{\text {incident }}\right|}\right\}=-20 \log \left\{\frac{4\left|\eta_{o}\right| \eta \mid}{\left|\eta_{o}+\eta\right|^{2}}\right\}+8.686 \alpha t
$$

For oblique incidence with incidence angle $\boldsymbol{\theta}_{\boldsymbol{i}}$ and transmitted angle $\boldsymbol{\theta}_{\boldsymbol{t}}$, two special cases are considered, one with the $\overrightarrow{\boldsymbol{E}}$ field perpendicular to the reflecting surface, and hence parallel to plane of incidence (parallel polarization, or TM wave), and the other is perpendicular polarization (TE wave), the analysis for reflection is more complicated. Therefore, perpendicularly approaching waves (TEM) were usually considered to provide a reasonable estimate of shielding effectiveness for different wave polarizations. In this paper, the transmitted coefficients for obliquely incident waves (TE or TM) are adopted for accurately comparing the shielding performance of superconductors with other materials against obliquely incident waves. The transmission coefficient for TM wave is denoted by $\boldsymbol{T}_{T M}$ and given by

$$
T_{T M}=\frac{2 \eta \cos \theta_{t}}{\eta \cos \theta_{t}+\eta_{o} \cos \theta_{i}}
$$

while for perpendicular polarization (TE wave), the reflection coefficient $\boldsymbol{T}_{\boldsymbol{T}}$ is given by

$$
T_{T E}=\frac{2 \eta \sec \theta_{t}}{\eta \sec \theta_{t}+\eta_{o} \sec \theta_{i}}
$$

Any other polarization may be considered as a linear combination of both TE and TM waves.

\section{B. Magnetic Shielding Effectiveness Calculations}


A magnetic shield is formed by a spherical shell has internal and external radii of $\boldsymbol{a}$ and $\boldsymbol{b}$; respectively. The relative permeability of the shell material is $\boldsymbol{\mu}_{\boldsymbol{r}}$, and the shell is immersed in an external uniform magnetic flux density $\overrightarrow{\boldsymbol{B}}_{\mathbf{1}}=\boldsymbol{B}_{\boldsymbol{o}} \hat{\boldsymbol{z}}$. To find the internal field $\overrightarrow{\boldsymbol{B}}_{\mathbf{3}}$ inside the hollow space $(\boldsymbol{r}<\boldsymbol{a})$, Laplace equations may be solved using the method of separation of variables in spherical coordinates $(r, \theta, \varphi)$ to give the following product solution [13] for the magnetic potential $\phi_{\boldsymbol{m i}}$ in the three regions of interest $\left(\phi_{m 1} \quad\right.$ for $r \geq b, \phi_{m 2} \quad$ for $a \leq r \leq b, \phi_{m 3} \quad$ for $\left.r \leq a\right)$ as $\phi_{m i}=\left(A_{i} r+C_{i} r^{-2}\right) \cos \theta$

The constants are determined from the boundary conditions, and hence the internal field $\overrightarrow{\boldsymbol{B}}_{\mathbf{3}}$ inside the hollow space $(\boldsymbol{r}<\boldsymbol{a})$ is obtained as

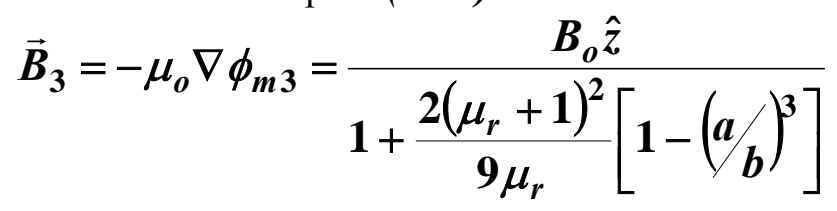

The magnetic shielding attenuation $\boldsymbol{S}$ is defined as the ratio of the applied external field $\boldsymbol{B}_{\boldsymbol{o}}$ to the internal $\boldsymbol{B}_{3}$, and given by

$$
S=\frac{B_{o}}{B_{3}}=1+\frac{2\left(\mu_{r}+1\right)^{2}}{9 \mu_{r}}\left[1-(a / b)^{3}\right]
$$

Usually the thickness of the shield $(\boldsymbol{t}=\boldsymbol{b}-\boldsymbol{a})$ is much less than its radius, then the magnetic shielding attenuation $S$ is approximately written as

$$
S \cong 1+\frac{2\left(\mu_{r}+1\right)^{2} t}{3 \mu_{r} b}
$$

For an idealized superconductor defined as a perfect diamagnetic material with magnetic susceptibility $\chi_{\boldsymbol{m}}=\mathbf{- 1}$, the superconducting spherical shell produces infinite value for $\boldsymbol{S}$, which yields zero magnetic flux density inside the superconductor (Meissner effect). As is evident from experimental observations, the concept of perfect flux exclusion is an idealization. Instead, a magnetic flux penetrates a thin layer at the surface of a superconductor and according to Maxwell - London equations, decreases exponentially as $\boldsymbol{B}(\boldsymbol{r})=\boldsymbol{B}(\mathbf{0}) e^{-r / \lambda_{L}}$

To solve the problem of a superconducting spherical shell placed in a uniform external applied field $\overrightarrow{\boldsymbol{B}}_{\mathbf{1}}=\boldsymbol{B}_{\boldsymbol{o}} \hat{\boldsymbol{z}}$, the fields must satisfy the following equations:

$\nabla \cdot \vec{B}=0, \quad \nabla \times \vec{H}=0, \quad \vec{B}=\mu_{o} \vec{H} \quad$ for the regions $r>b$, and $r<a$ $\nabla \cdot \vec{B}=0, \quad \nabla^{2} \vec{B}=\left(1 / \lambda_{L}^{2}\right) \vec{B} \quad$ within the superconducting shell $a<r<b$

The fields $\overrightarrow{\boldsymbol{B}}_{\mathbf{1}}$ and $\overrightarrow{\boldsymbol{B}}_{\mathbf{3}}$ in the regions $\boldsymbol{r}>\boldsymbol{b}$, and $\boldsymbol{r}<\boldsymbol{a}$; respectively, are obtained from magnetic scalar potentials $\phi_{m \mathbf{1}}$ and $\phi_{m \mathbf{3}}$ satisfying Laplace equation and the boundary 
conditions imposed at $\boldsymbol{r} \rightarrow \infty, \boldsymbol{r}=\boldsymbol{b}, \boldsymbol{r}=\boldsymbol{a}$, and $\boldsymbol{r}=\mathbf{0}$, while for the field within the superconducting shell, the vector Helmholtz equation $\nabla^{2} \overrightarrow{\boldsymbol{B}}_{2}=\left(1 / \lambda_{L}{ }^{2}\right) \overrightarrow{\boldsymbol{B}}_{2}$ must be firstly solved to obtain $\overrightarrow{\boldsymbol{B}}_{\mathbf{2}}(\boldsymbol{r}, \theta)$ in the region $\boldsymbol{a}<\boldsymbol{r}<\boldsymbol{b}$. From the form of $\overrightarrow{\boldsymbol{B}}_{\mathbf{1}}=\boldsymbol{B}_{\boldsymbol{o}} \hat{\boldsymbol{z}}$, and the matched boundary conditions at $\boldsymbol{r}=\boldsymbol{b}$, the following solution for $\overrightarrow{\boldsymbol{B}}_{2}(\boldsymbol{r}, \theta)$ may be assumed as $\overrightarrow{\boldsymbol{B}}_{2}(\boldsymbol{r}, \theta)=\boldsymbol{B}_{r} \hat{\boldsymbol{r}}+\boldsymbol{B}_{\theta} \hat{\boldsymbol{\theta}}$ where

$$
B_{r}(r, \theta)=f_{1}(r) \cos \theta, \quad B_{\theta}(r, \theta)=f_{2}(r) \cos \theta
$$

The two functions $f_{1}(r)$ and $f_{2}(r)$ must be determined so that $\nabla^{2} \vec{B}_{2}=\left(1 / \lambda_{L}{ }^{2}\right) \vec{B}_{2}$ and the boundary conditions at $\boldsymbol{r}=\boldsymbol{a}$, and $\boldsymbol{r}=\boldsymbol{b}$ are satisfied. The complete set of boundary conditions are then applied on the proposed forms of fields in the three regions to yield the approximate solutions $\left(\boldsymbol{a}>>\lambda_{\boldsymbol{L}}\right)$ for magnetic flux density in each region as:

$$
\begin{aligned}
\vec{B}_{1} & =\hat{r} B_{o} \cos \theta\left[1-(b / r)^{3}\right]-\hat{\theta} B_{o} \sin \theta\left[1+\frac{1}{2}(b / r)^{3}\right] \\
\vec{B}_{2} & =\frac{3 B_{o} b \lambda_{L} \cos \theta}{r^{2} \sinh \left(\frac{b-a}{\lambda_{L}}\right)}\left[\hat{r} \cosh \left(\frac{r-a}{\lambda_{L}}\right)+\hat{\theta} \frac{\tan \theta}{2}\left(\cosh \left(\frac{r-a}{\lambda_{L}}\right)+\frac{r}{\lambda_{L}} \sinh \left(\frac{b-a}{\lambda_{L}}\right)\right)\right] \\
\vec{B}_{3} & =\frac{3 \lambda_{L} B_{o}}{b \sinh \left(\frac{b-a}{\lambda_{L}}\right)}(\hat{r} \cos \theta-\hat{\theta} \sin \theta)
\end{aligned}
$$

The magnetic shielding attenuation factor $\boldsymbol{S}^{\boldsymbol{s c}}$ for the superconducting spherical shell which is characterized by London penetration depth $\lambda_{\boldsymbol{L}}$, inner radius $\boldsymbol{a}$, and outer radius $\boldsymbol{b}$ is then obtained as

$$
S^{s c}=S_{r}=S_{\theta}=\frac{B_{o}}{B_{3}}=\frac{b}{3 \lambda_{L}} \sinh \left(\frac{b-a}{\lambda_{L}}\right)
$$

\section{NUMERICAL CALCULATIONS AND DISCUSSIONS}

To validate the developed model, the superconducting alloy $\left(\boldsymbol{N} \boldsymbol{b}_{30}-\boldsymbol{T a} \boldsymbol{a}_{70}\right)$ is chosen with the parameters: $T_{c}=6 K, \quad \sigma_{o}=2 \times 10^{7} \Omega^{-1} m^{-1}$, and $\lambda_{L}(0)=90 \mathrm{~nm}$. The variation of electromagnetic properties versus the normalized temperature $\left(\boldsymbol{T} / \boldsymbol{T}_{c}\right)$ is investigated at frequency $\boldsymbol{f}=\mathbf{9} \mathbf{G H z}$, and plotted in Fig.1 for superconducting and normal - state carrier densities, while London penetration depth is shown in Fig.2. The complex conductivity and permittivity are shown in figures 3 and 4; respectively. The surface impedance is then calculated and plotted in Fig. 5, where excellent agreement is obtained between the calculated 
surface resistance $\boldsymbol{R}_{\boldsymbol{s}}$ by the developed model and the measured results of [12]. The high $\boldsymbol{T}_{\boldsymbol{c}}$ superconductor compound; Yttrium-Barium-copper oxide (YBCO), with $\boldsymbol{T}_{\boldsymbol{c}}=90 \mathrm{~K}$ is then modeled at $\boldsymbol{f}=\mathbf{1 9} \mathbf{G H z}$, and the results are displayed in figures $6: 9$, where the temperature variation of superconducting carrier density ( $\boldsymbol{N}_{S}$ or $\boldsymbol{n}_{\mathrm{s}}$ ) and normal (conduction) carrier density $\left(\boldsymbol{N}_{c}\right.$ or $\left.\boldsymbol{n}_{\boldsymbol{n}}\right)$ are shown in Fig.6, while Fig.7 shows the variation of London penetration depth $\lambda_{L}$ versus temperature $\boldsymbol{T}$. The surface resistance $\boldsymbol{R}_{\boldsymbol{s}}$ and reactance $\boldsymbol{X}_{\boldsymbol{s}}$ are then calculated, plotted versus $\boldsymbol{T}$, and compared satisfactorily with the results of reference [11] in figures 8 and 9; respectively. The real and imaginary parts of relative complex permittivity $\varepsilon_{r}=\varepsilon^{\prime}-j \varepsilon^{\prime \prime}=\varepsilon_{r 1}-j \varepsilon_{r 2} \quad$ for $\quad$ Niobium $\left(\lambda_{L}(0)=85 \mathrm{~nm}, T_{c}=10 \mathrm{~K}, \sigma_{o}(100 \mathrm{~K})=0.3 e^{8} \mathrm{~S} / \mathrm{m}\right)$ are also calculated, plotted versus temperature, and found in exact agreement with those of reference [9], as shown in Fig.10 at $\boldsymbol{f}=10 \mathrm{GH} \boldsymbol{z}$. The variation of real and imaginary parts of the relative complex permittivity versus frequency are also plotted in Fig.11. The shielding properties of different YBCO superconductor structures are then investigated. First, a slab of YBCO with thickness $\boldsymbol{t}=\mathbf{0 . 5} \boldsymbol{\mu m}$ is placed in free space and subjected to an incident plane wave at $\boldsymbol{f}=10 \mathrm{GHz}$. The variation of shielding coefficients versus $\boldsymbol{T}$ due to reflection and absorption of TEM wave are shown in Fig.12, while the TEM reflection shielding coefficient is compared with TE, and TM reflection shielding coefficients at $\boldsymbol{\theta}_{\boldsymbol{i}}=\mathbf{4 5}^{\boldsymbol{\sigma}}$ in Fig.13. Second, the magnetic shielding coefficients for YBCO and high permeability ferrous material $\left(\boldsymbol{\mu}_{r}=20000\right)$ spheres (inner radius $=\boldsymbol{a}=49 \boldsymbol{\mu m}$, and outer radius $=\boldsymbol{b}=\mathbf{4 9 . 5} \boldsymbol{\mu m}$ ) are plotted versus $\boldsymbol{T}$, and compared in Fig. 14. When the same parameters are used, but at different (lower) frequency $\boldsymbol{f}=\mathbf{5 0} \mathbf{H z}$, the shielding coefficients for the superconducting slab have been greatly improved, due to the obtained higher conductivity for the superconducting slab at the lower frequency [14], as shown in figures 15 and 16 compared with those of figures 12 and 13. Finally, the same parameters are used, but the inner radius is increased to be $\mathbf{1 ~} \mathbf{~ c m}$, keeping the same thickness $\boldsymbol{t}=0.5 \boldsymbol{\mu m}$, the shielding properties for the superconducting slab at $\boldsymbol{f}=10 \mathrm{GHz}$ are found to be the same as those in figures 12 and 13, while the magnetic shielding properties for both superconductor and ferrous spheres are found strongly dependent on the sphere radii as shown in Fig.17, where the superconductor sphere achieved shielding coefficient $S \sim 10^{5}$ compared with $\boldsymbol{S} \sim 2$ for the same sphere, but made from ferrous material having $\boldsymbol{\mu}_{r}=\mathbf{2 0 0 0 0}$.

\section{CONCLUSION}

An improved model has been developed to investigate the shielding properties of different superconducting structures. The proposed model incorporates the two-fluid model, BCS theory and GL theory with Maxwell equations and experimental Gorter-Casmir relations. The developed algorithm has been used to model different low and high $\boldsymbol{T}_{\boldsymbol{c}}$ superconductors, and the results have been found in excellent agreement with both published theoretical and measured results as shown in figures $5,8,9$, and 10 . Different superconducting shielding structures have been analyzed. YBCO slab with thickness $t=0.5 \mu m \cong 3 \lambda_{L}(0)$ has exhibited very high shielding coefficients against TEM wave at $\boldsymbol{f}=\mathbf{5 0} \mathbf{H z}$, where $\boldsymbol{S}=\mathbf{5 9 5}$ : $540 \boldsymbol{d B}$ versus $\boldsymbol{T}$ as shown in Fig.15. When frequency is increased to $\boldsymbol{f}=\mathbf{1 0} \mathbf{G H z}$, YBCO conductivity is degraded [14], and hence TEM shielding effectiveness is lowered to $\boldsymbol{S}=\mathbf{2 1 5}$ : $150 \boldsymbol{d B}$ versus $\mathrm{T}$ as shown in Fig.12. Similar behavior has been obtained for shielding effectiveness due to reflection against incident TE and TM waves as shown in figures 13 and 
16. The shielding properties of YBCO sphere $\left(\boldsymbol{t}=\mathbf{0 . 5} \boldsymbol{\mu m} \cong \mathbf{3} \lambda_{L} \mathbf{( 0 )}\right)$ against static magnetic field has been investigated and compared with those for the same sphere, but made from high $-\mu$ alloy having $\boldsymbol{\mu}_{r}=\mathbf{2 0 0 0 0}$. When the inner radius was chosen impractically small; $\boldsymbol{a}=49 \boldsymbol{\mu m}$, the high- $\mu$ sphere exhibited $S=140$ compared with $\boldsymbol{S} \sim \mathbf{1 0 0 0}$ for YBCO sphere at $\boldsymbol{T}=\boldsymbol{0}: 0.3 \boldsymbol{T}_{\boldsymbol{c}}$ as shown in Fig.14. Keeping the thickness $\boldsymbol{t}$ constant, and increasing the inner radius to be practical as $\boldsymbol{a}=\mathbf{1} \mathbf{c m}$, the shielding coefficient for the high- $\mu$ sphere has been greatly reduced to be $S \sim 2$, compared with $S \sim 2 \times 10^{5}: 1000$ versus T for YBCO sphere as shown in Fig.17, which strongly recommend the superconducting structure for perfect shielding.

\section{REFERENCES}

[1] A. Bourdillon, and N. X. Bourdillon, 'High Temperature Superconductors: Processing and Science," Academic Press, Inc., New York, 1994.

[2] J. T. Williams and S. A. Long, "High Temperature Superconductors and Their Applications in Passive Antenna Systems,' IEEE Antennas and Propagation Magazine, pp. 718, Aug. 1990.

[3] C. Rose and M. J. Gans, 'A Dielectric-Free Superconducting Coaxial Cable,' IEEE Trans. on Microwave Theory and Techniques, Vol. 38, No. 2, pp. 166-177, Feb. 1990.

[4] J. O. Willis, M. E. McHenry, M. P. Maley, and H. Sheinberg, 'Magnetic Shielding by Superconducting Y-Ba-Cu-O Hollow Cylinders,' IEEE Trans. on Magnetics, Vol. 25, No. 2, pp. 2502-2505, March 1989.

[5] G. Shulz, 'Shielding Theory and Practice,' IEEE Trans. on Electromagnetic Compatibility, Vol. 30, pp. 211-221, 1988.

[6] T. P. Orlando and K. A. Delin, 'Foundations of Applied Superconductivity,' AddisonWesley Publishing Company, 1991.

[7] F. J. Owens and C. P. Poole, 'Electromagnetic Absorption in the Copper Oxide Superconductors,' Kluwer Acdemic/Plenum Publishers, New York, 1999.

[8] A. A. Hussein and M. Sayer, 'Simple Magnetic Shielding Experiment on high $T_{c}$ Superconducting Bulk Ceramics and Thin Films, Metals, and Alloys,' Cryogenics, Vol. 32, No.1, pp. 64-68, 1992.

[9] K. K. Mei and G. C. liang, 'Electromagnetics of Superconductors,' IEEE Trans. on Microwave Theory and Techniques, Vol. 39, No. 9, pp. 1545-1552, 1991.

[10] E. C. Jordan and K. G. Balmain, 'Electromagnetic Waves and Radiating Systems,' Prentice-Hall, Inc., 1982.

[11] O. G. Vendik, I. B. Vendik, and D. I. Kaparkov, 'Emperical Model of the Microwave Properties of High-Temperature Superconductors,' IEEE Trans. on Microwave Theory and Techniques, Vol. 46, No. 5, pp. 469-478, May 1998.

[12] C. M. Lyneis and J. P. Turneaure, 'Measurement of the Microwave Properties of Superconducting $\mathrm{Nb}_{30}$-Ta 70 , EEE Trans. on Magnetics, Vol. 13, No. 1, January 1977.

[13] M. A. Plonus, 'Applied Electromagnetics,' Mcgraw-Hill, Inc., 1987.

[14] M. Majoros, B. A. Glowacki, A. M. Campbell, G. A. Levin, P. N. Barnes, and M. Polak, 'AC Losses in Striated YBCO Coated Conductors', IEEE Transactions on Applied Superconductivity, 15 (2) 2819-2822 (June 2005). 

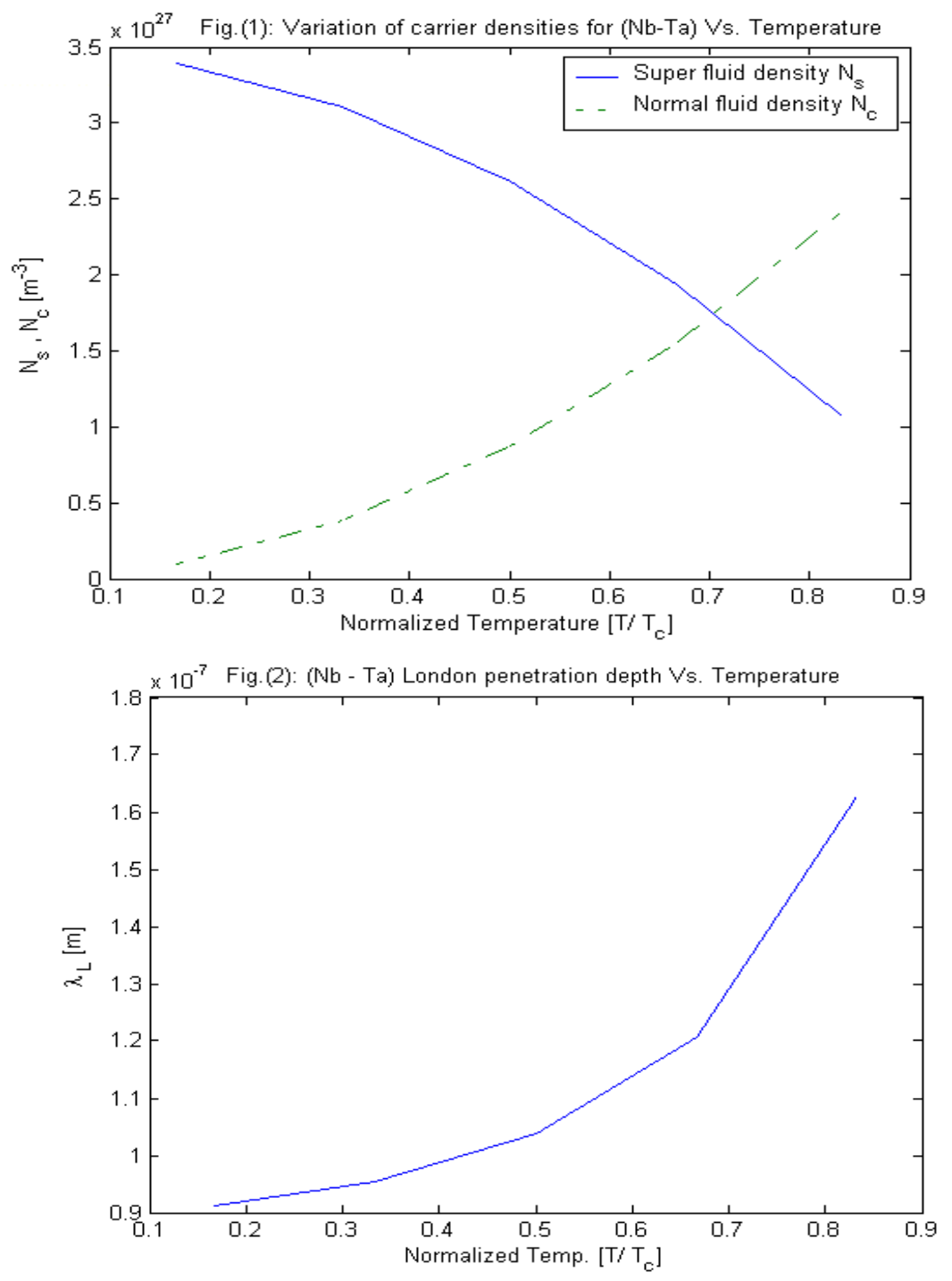
Proceedings of the $5^{\text {th }}$ ICEENG Conference, 16-18 May, 2006

$\mathrm{AW}-3-11$
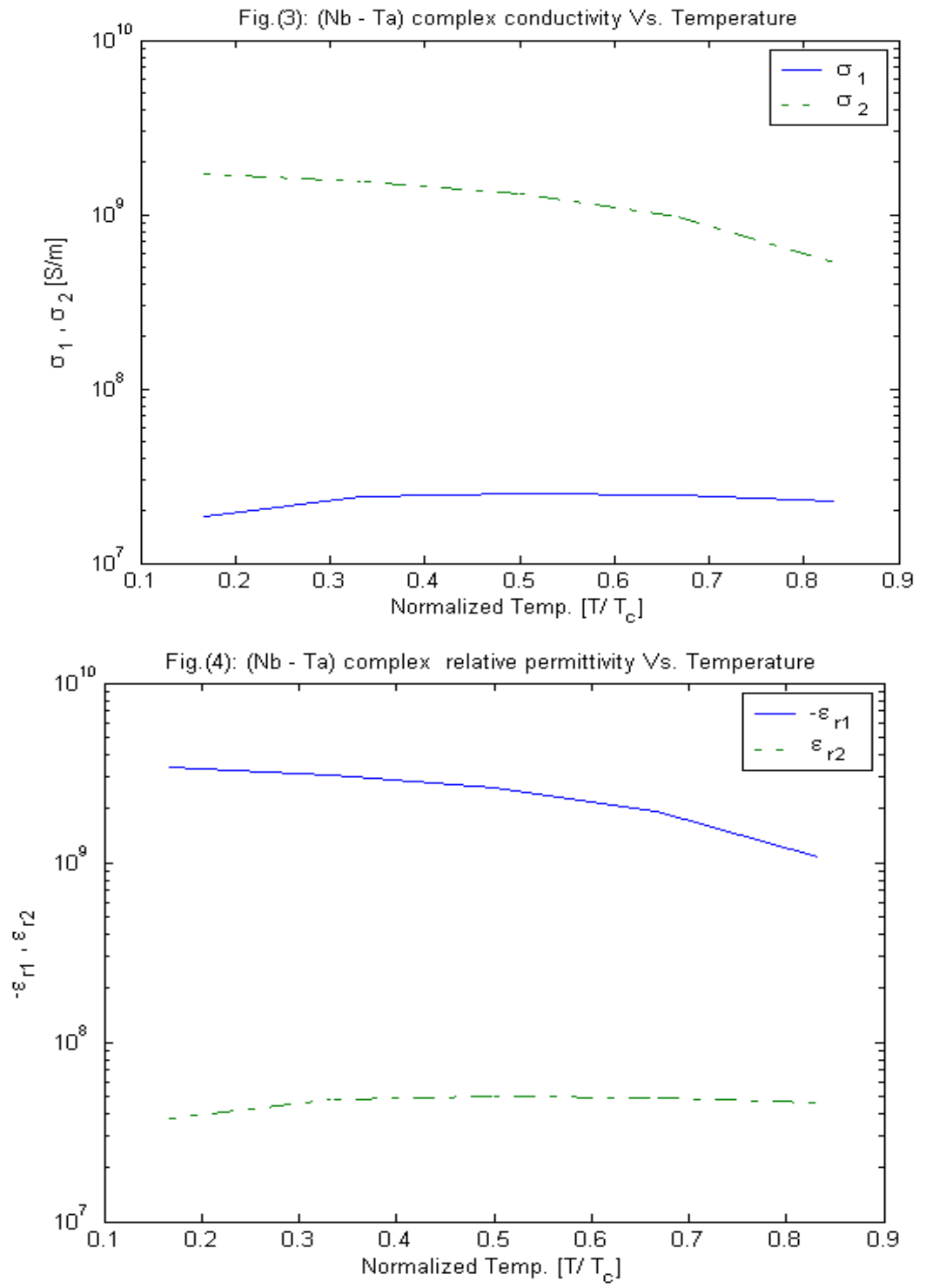
Proceedings of the $5^{\text {th }}$ ICEENG Conference, 16-18 May, 2006
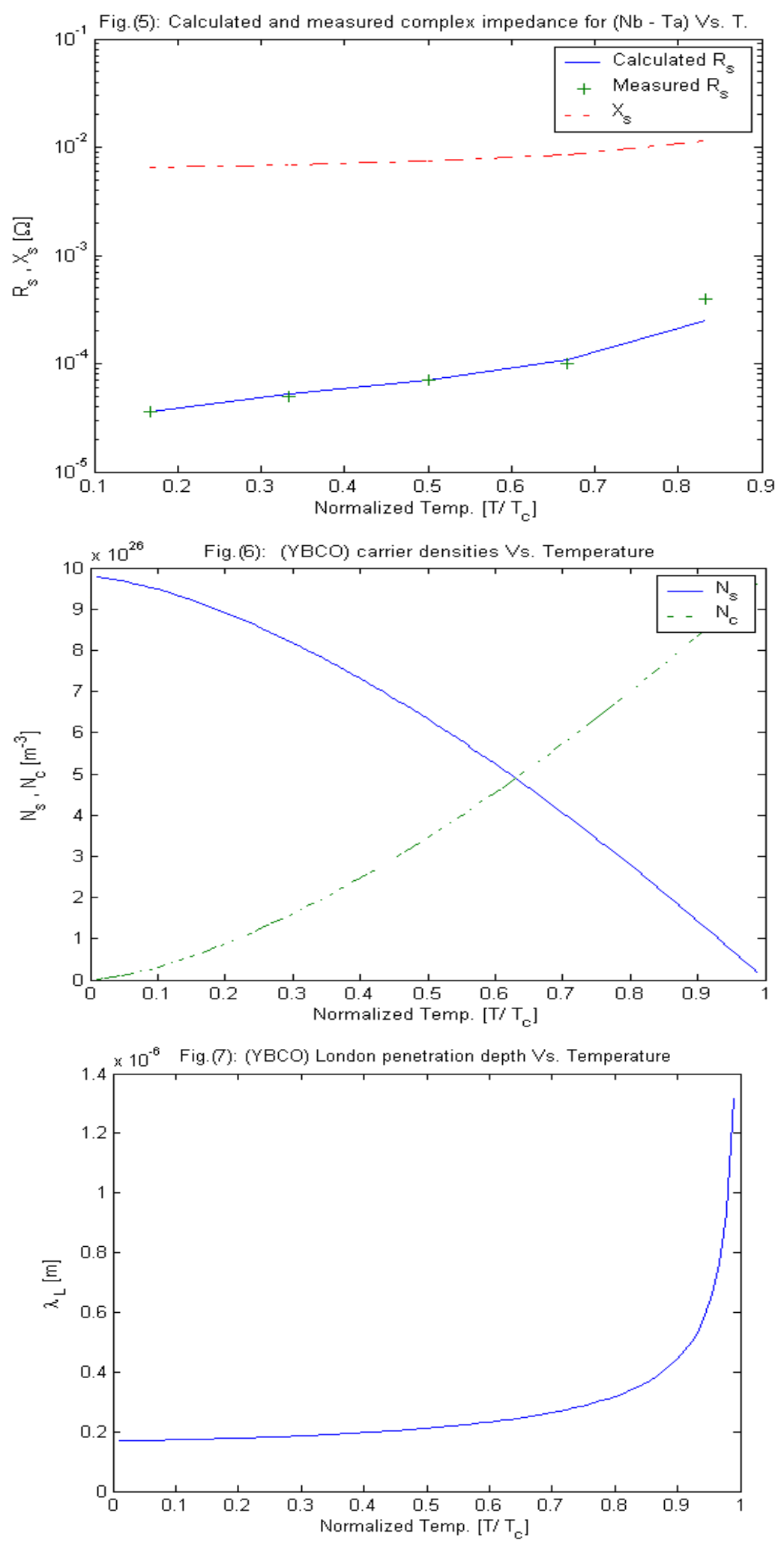
Proceedings of the $5^{\text {th }}$ ICEENG Conference, 16-18 May, 2006

$\mathrm{AW}-3-14$
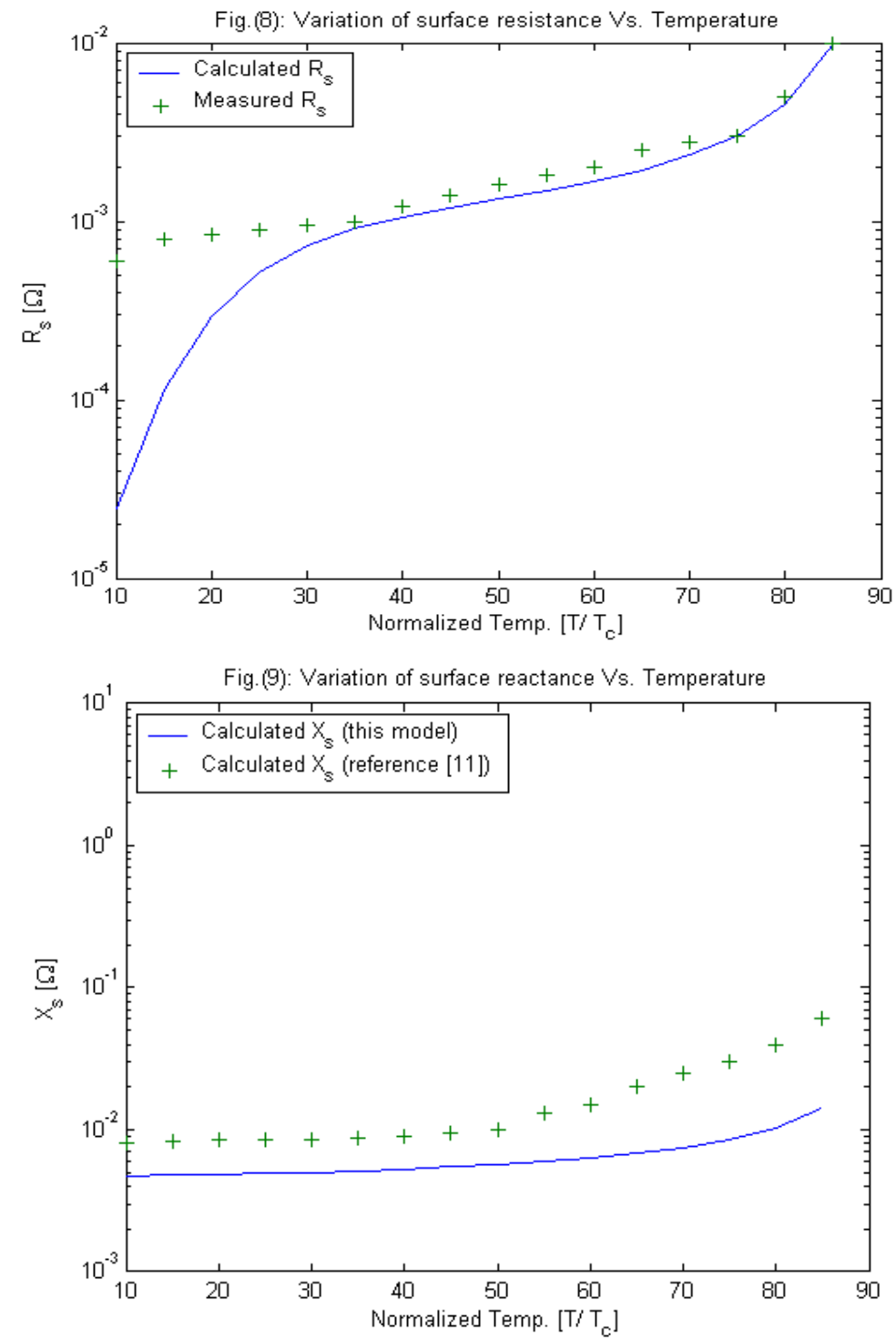

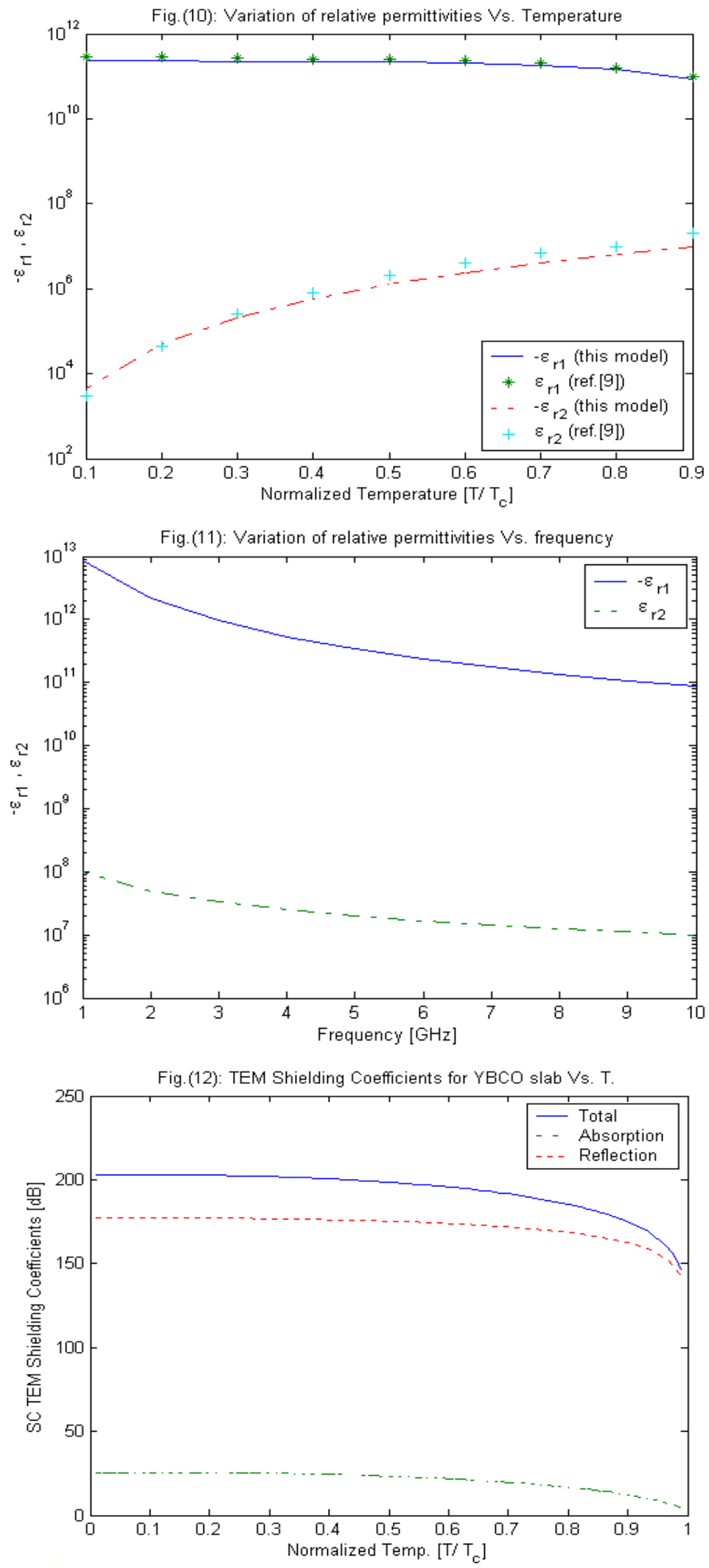
Proceedings of the $5^{\text {th }}$ ICEENG Conference, 16-18 May, 2006

$\mathrm{AW}-3-10$
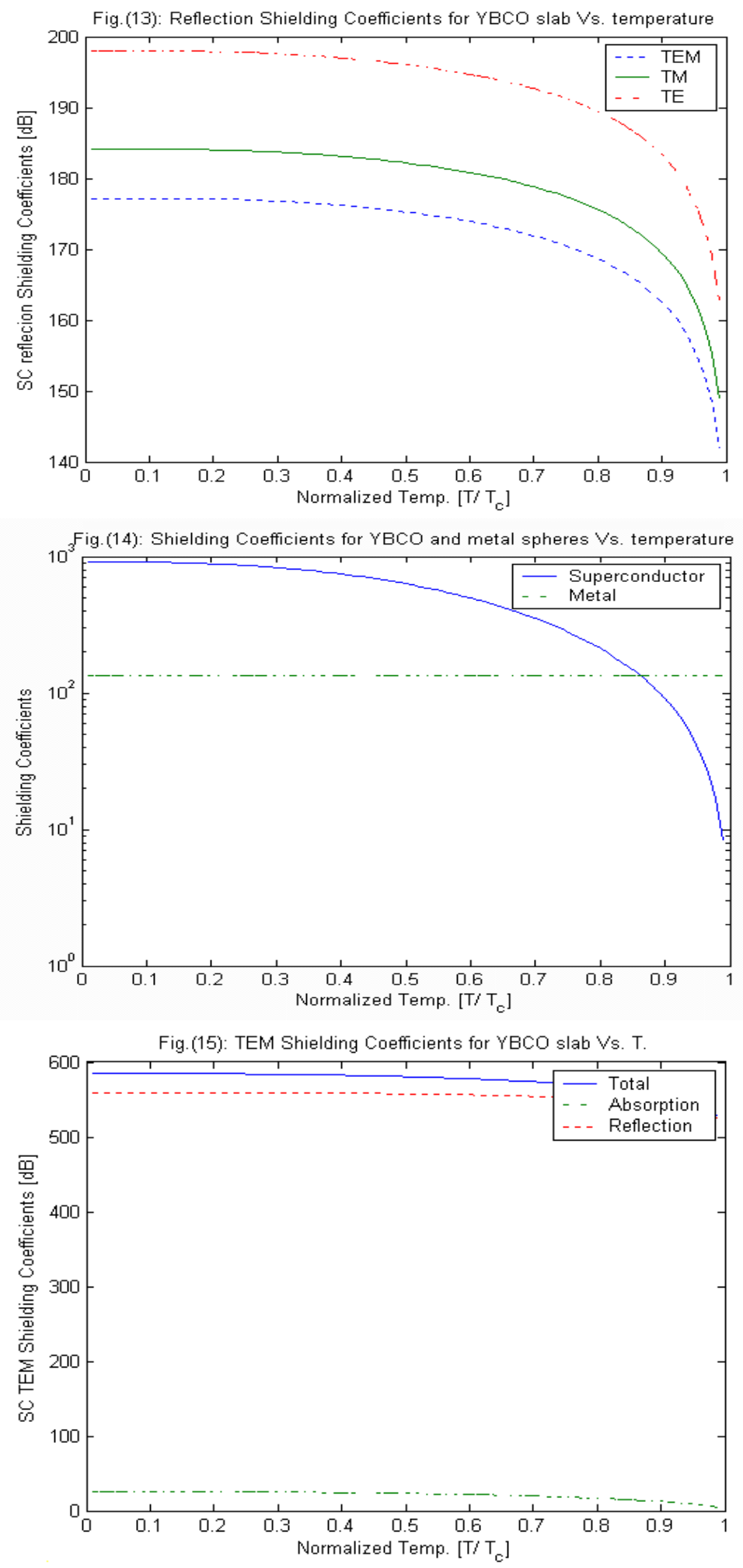

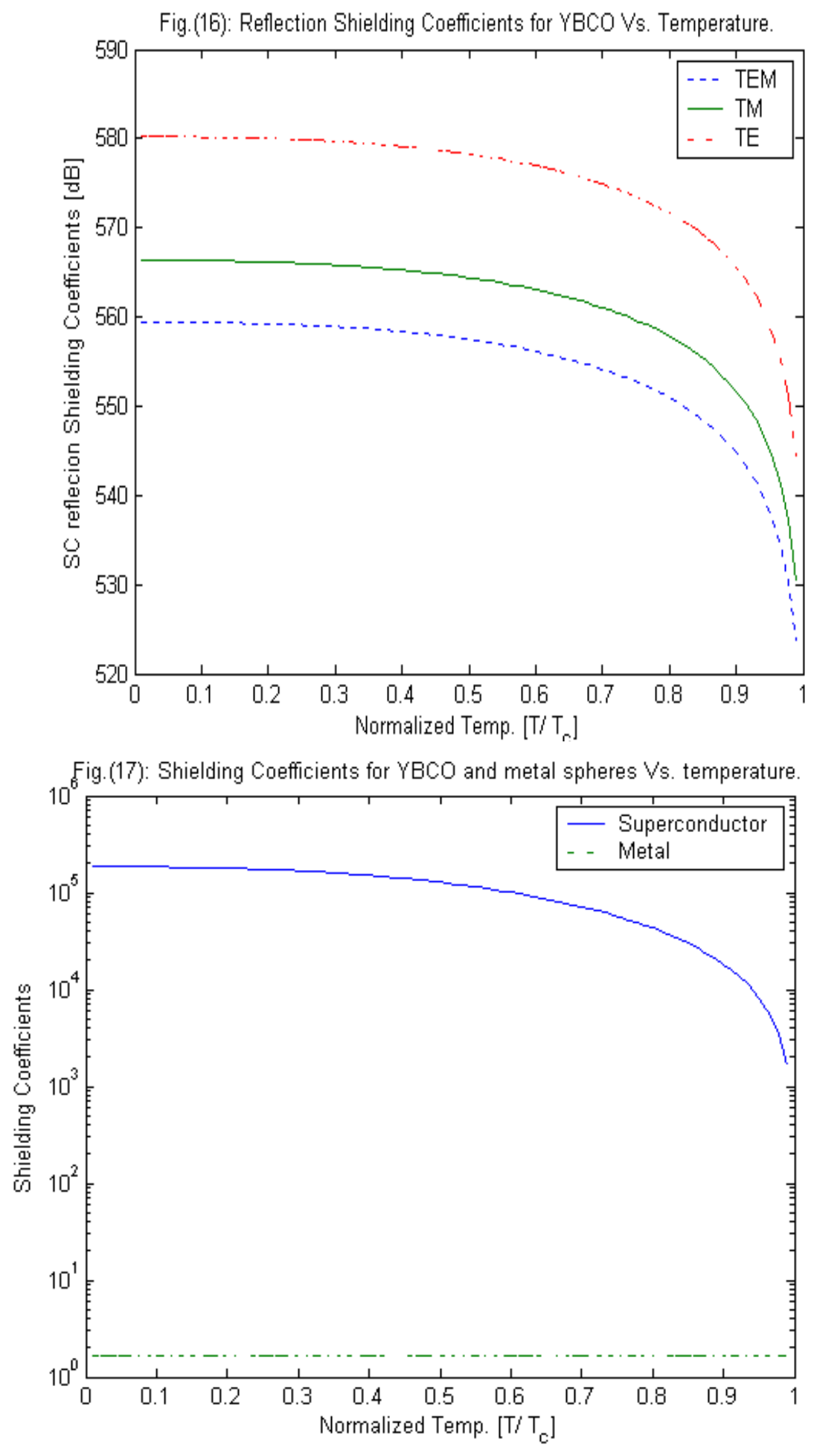